\title{
Congenital thrombocytopenia associated with GNE mutations in twin sisters: a case report and literature review
}

\author{
Li Xin ${ }^{1,2}$, Ying Li', Min Lei ${ }^{1}$, Jing Tian¹, Zuocheng Yang ${ }^{1}$, Shoujin Kuang ${ }^{1}$, Yanjuan Tan ${ }^{1}$ and Tao Bo ${ }^{1 *} \mathbb{D}$
}

\begin{abstract}
Background: Neonatal thrombocytopenia is common in preterm and term neonates admitted to neonatal intensive care units. The etiology behind neonatal thrombocytopenia is complex. Inherited thrombocytopenia is rare and usually results from genetic mutations.
\end{abstract}

Case presentation: Here we report a case of twins with severe inherited thrombocytopenia presented in the neonatal period who were shown to be compound heterozygotes for 2 UDP-N-acetylglucosamine 2-epimerase (GNE) gene mutations, C.1351C > T and C.1330G > T, of which c.1330G > T is a novel mutation.

Conclusion: These two GNE mutations may help in the diagnosis and management of thrombocytopenia diagnosed in neonates.

Keywords: Congenital thrombocytopenia, UDP-N-acetyl-glucosamine 2-epimerase/N-acetylmannosamine kinase (GNE), Sialic acid, Neonates, Twins

\section{Background}

Thrombocytopenia is defined as a platelet count $<150 \times$ $10^{9} / \mathrm{L}$ and is the most frequent hematological disorder observed in neonates admitted to the neonatal intensive care unit (NICU). This disorder has an incidence between 18 and 35\% [1] It is known that reduced production of platelets induces thrombocytopenia, whose heritable forms are frequently caused by genes that regulate megakaryocytic differentiation and/or platelet production [2] With the development of diagnostic methods, neonatal thrombocytopenia, especially the congenital type, was linked to genetic mutations.

UDP-N-acetyl-glucosamine 2-epimerase/N-acetylmannosamine kinase (GNE), a key enzyme of sialic acid biosynthesis, initiates and regulates the biosynthesis of $\mathrm{N}$ acetylneuraminic acid [3] Mutations in GNE have been

\footnotetext{
* Correspondence: boily@yeah.net

'Department of Pediatrics, The Third Xiangya Hospital, Central South University, Tongzipo Road NO.138, Changsha 410013, China

Full list of author information is available at the end of the article
}

identified in both hereditary inclusion body myopathy and Nonaka myopathy, which is also termed as distal myopathy with rimmed vacuoles (DMRV; MIM\#605820) [2, 4] In recent years, the relationship between GNE mutations and congenital thrombocytopenia has been reported $[3,5-8]$ Here, we report a case of twin sisters who were shown to have novel combined heterozygous GNE mutations and diagnosed with refractory thrombocytopenia.

\section{Case report}

A 22-day-old female newborn exhibited a platelet count of $1 \times 10^{9} / \mathrm{L}$ and showed detectable traces of blood in her stools. At the same time, her twin sister also had a platelet count of $6 \times 10^{9} / \mathrm{L}$. The twins were born $373 / 7$ weeks of gestational through cesarean section to a mother who denies unfavorable obstetric or medical histories. Moreover, there was no history of thrombocytopenia in the family. When the newborns were admitted to the NICU, vital signs and physical examinations appeared normal. Blood laboratory studies 
showed severe thrombocytopenia and mild anemia in the twins. The size of their peripheral blood platelets was shown to range from normal to large and their mean platelet volume was 10.6-13.2 fl (reference range: 6.5-12 fl). Routine urine, stool, liver function and renal function tests all appeared as normal. TORCH- and platelet-associated antibodies were negative. Megakaryocytes in the bone marrow showed normal morphology, but their maturation was hindered. Whole exon sequencing (WES) analysis revealed novel compound heterozygous mutations in exon 6 of the GNE gene (Fig. 1a), including c. $1351 \mathrm{C}>\mathrm{T}$, (p. Arg451*) and c.1330G > T, (p. Asp444Tyr) (Fig. 1b, c). No other gene mutations related to thrombocytopenia were identified. A genogram directly confirmed that the twins were compound heterozygotes for these mutations (Fig. 1d). In the Genome Aggregation Database (gnomAD), the occurrence frequency of the two mutations was found to be $4 / 251452$ $($ c.1351C $>$ T) and $1 / 251452$ (c.1330G $>$ T). The c. 1330G > T was found to be a novel mutation. Despite being reported in HGMD as described by Tomimitsu [9] et al and Mori-Yoshimura [10] et al, c.1351C > T mutations associated with thrombocytopenia are first reported here. The p. Arg $451^{*}$ based reference sequence NM_005476.6 is a non-sense mutation. Furthermore, mutation pathogenicity prediction software, including Sorting Intolerant From Tolerant (SIFT), Polyphen2, LRT, MutationTaster, PredictSNP (https://loschmidt. chemi.muni.cz/predictsnp/) and FATHMM, predicted that the c.1330G > T mutation was harmful and destabilizing. Homology modeling was performed for the wildtype or mutant proteins using the SWISS-MODEL server (https://swissmodel.expasy.org/). As shown in Fig. 1e, c.1351C $>\mathrm{T}$ results in the deletion of 8 amino acids starting at position 451, whereas c.1330G $>\mathrm{T}$ results in an Asp to Tyr mutation at position 444. Therefore, the twins were diagnosed with congenital thrombocytopenia associated with GNE mutations.

The twins received platelet and intravenous immunoglobulin (IVIG) transfusions soon after admission to the NICU given their severe thrombocytopenia, but their baseline platelet counts remained as less than $10 \times 10^{9} / \mathrm{L}$. After a 3-month follow-up, the twins did not show bleeding or signs of muscle wasting.

\section{Discussion and conclusion}

The human GNE gene (NM_001128227.2), localized on chromosome 9p13.3, consists of 14 exons and encodes 724 amino acids $[3,5,6,11]$ The protein encoded by this gene is a bifunctional enzyme that initiates and regulates the biosynthesis of $\mathrm{N}$-acetylneuraminic acid (NeuAc), which is a precursor of sialic acid [12].

Mutations in the GNE gene decreases its enzymatic activity and affects the two initial steps in sialic acid biosynthesis. As a result, sialic acid production decreases and consequently, sialylation, or the incorporation of sialic acid to glycoproteins and glycolipids, also decreases [13] Hyposialylation was previously identified in mouse models of GNE myopathy [14] More than 10 GNE gene mutations have been reported in human DMRV. However, the exact mechanisms behind GNE defects leading to DMRV are still not fully understood.

As a major determinant of cell surface sialylation in human hematopoietic cell lines and a critical regulator of cell surface adhesion molecules [15], GNE is also expressed in bone marrow. Sialic acid is known to play significant roles in platelet functions and is located in the platelet membrane, forming during glycosylation. Several groups have shown that platelets deficient in sialylation are removed from circulation, resulting in shortened circulation lifetimes and thrombocytopenia [16]

In 2014, two families diagnosed with DMRV associated with thrombocytopenia were reported. Izumi et al [2] reported that two adult siblings diagnosed with DMRV and thrombocytopenia harbored two compound heterozygous GNE mutations, p.V603L and p.G739S. Zhen et al [17] reported that two adult siblings in a DMRV family exhibited thrombocytopenia and showed compound heterozygous GNE mutations including a missense mutation c.649 T > C, (p.Y217H) and a frameshift mutation c.1543-1544 del GA, (p.D515Qfs*2). These patients showed mild-tomoderate thrombocytopenia and no obvious bleeding.

In 2018, it was reported that GNE mutations cause thrombocytopenia without associated myopathy. Futterer et al [8] reported that two cousins in a consanguineous family manifested moderate to severe bleeding associated with severe thrombocytopenia and required regular platelet transfusions. In addition, homozygous mutations c. $1246 \mathrm{G}>\mathrm{A}$, (p. G416r) in the GNE gene were detected. Revel-Vilk et al [18] summarized nine patients from three families all exhibiting thrombocytopenia and mild to moderate hemorrhagic tendencies. All of these individuals contained compound homozygous or heterozygous mutations in GNE, of which eight of the nine patients in showed no evidence of myopathy and one patient with neuromuscular symptoms showed a muscle biopsy inconsistent with GNE myopathy.

In the case of the twins described here, refractory severe thrombocytopenia was identified in the neonatal period and two novel compound heterozygous GNE mutations, p.Arg $451^{*}$ and p.Asp444Tyr, were detected using wholeexome sequencing. Compared with other reports, these twins are some of the youngest reported and their baseline platelet counts were of the lowest values when they were diagnosed. Consistent with other reports [17, 19, 20], mutations in GNE associated with thrombocytopenia were within the $\mathrm{N}$-acetylmannosamine (ManNAc) kinase domains of the GNE protein. Thus, this domain may be an 

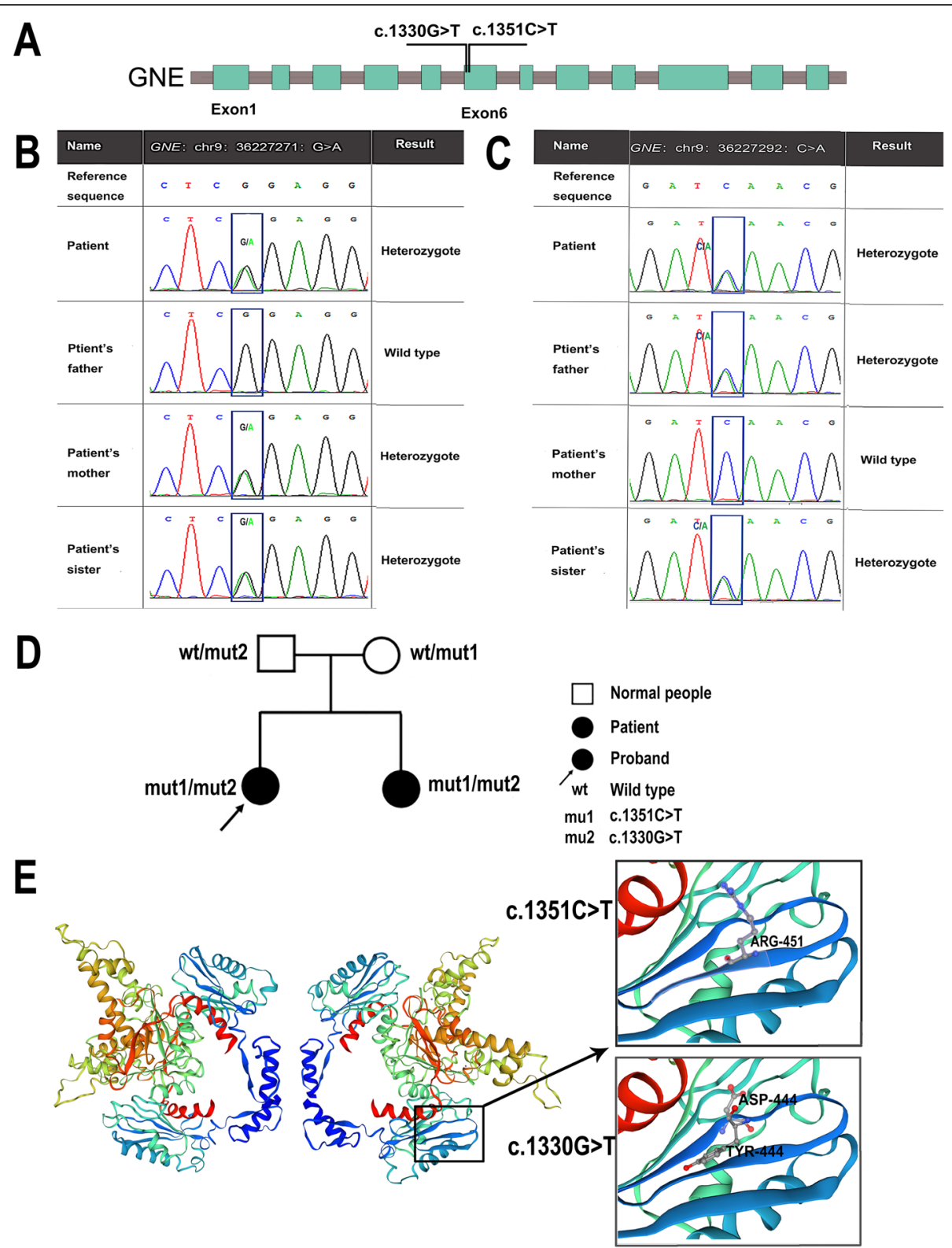

Fig. 1 Sequencing and a genogram of the Trio. a:Schematic diagram of GNE exons. Mutation positions were labeled with black lines. b: The c.1351C > T mutation. This mutation is a non-sense mutation, where arginine (Arg) mutates to a termination codon, which is inherited from the mother. c: The c.1330G > T mutation. This mutation is inherited from the father and is a missense mutation, resulting when aspartic acid (Asp) mutates to tyrosine (Tyr). $\mathbf{d}$ : Parents are carriers of each mutation and show no phenotypes. The twins simultaneously inherited two mutations from their parents and presented with refractory thrombocytopenia several days after birth. e: 3D homology model changes induced by the c. $1330 \mathrm{G}>\mathrm{T}$ and $\mathrm{C} .1351 \mathrm{C}>\mathrm{T}$ mutations

interesting site for further investigation related to congenital thrombocytopenia associated with GNE mutations.

In summary, in this report, we describe twins with two novel compound heterozygous GNE mutations, including p.Arg451* and p.Asp444Tyr, who manifested only severe thrombocytopenia without myopathy. Yet the role of GNE protein in the synthesis and functions of platelet requires further investigation.

\section{Abbreviations}

GNE: UDP-N-acetyl-glucosamine 2-epimerase/N-acetylmannosamine kinase; NeuAc: N-acetylneuraminic acid; ExAC: Exome Aggregation Consortium; gnomAD: Genome Aggregation Database; SIFT: Sorting Intolerant From Tolerant

\section{Acknowledgments}

We first thank the patients and their parents. We are also grateful to CIPHER GENE company, providing us the whole-exome sequencing and data analysis. 


\section{Authors' contributions}

YL, ML, JT, ZCY, SJK, YJT participated in the management of the patients, acquired the clinical data, and prepared the samples from the family members. XL, TB drafted the initial manuscript, and reviewed and revised the manuscript. All authors approved the final manuscript as submitted and agree to be accountable for all aspects of the work.

\section{Funding}

No funding was received.

\section{Availability of data and materials}

The datasets analysed during the current study are available in the 360 medical repository(https://yunpan.360.cn/surl_yPErJZuASnH). NM_005476.6(genebank https://www.ncbi.nlm.nih.gov/nuccore/NM_005476.6

NM_001128227.2(genebank https://www.ncbi.nlm.nih.gov/nuccore/NM_ $001128227.2)$.

\section{Ethics approval and consent to participate}

This study was approved by the Ethics Committee of the 3rd Xiangya Hospital, Central South University. Written informed consent was obtained from the parents of the patient.

\section{Consent for publication}

Written informed consent was obtained from the parents for publication of this case report.

\section{Competing interests}

The authors have no conflicts of interest to disclose.

\section{Author details}

'Department of Pediatrics, The Third Xiangya Hospital, Central South University, Tongzipo Road NO.138, Changsha 410013, China. ${ }^{2}$ Chengdu Women's and Children's Central Hospital, School of Medicine, University of Electronic Science and Technology of China, Chengdu 611731, Sichuan, China.

Received: 5 September 2019 Accepted: 4 November 2020

Published online: 16 November 2020

\section{References}

1. Cremer M, Sallmon H, Kling PJ, Buhrer C, Dame C. Thrombocytopenia and platelet transfusion in the neonate. Semin Fetal Neonatal Med. 2016;21(1): $10-8$.

2. Izumi R, Niihori T, Suzuki N, Sasahara Y, Rikiishi T, Nishiyama A, et al. GNE myopathy associated with congenital thrombocytopenia: a report of two siblings. Neuromuscul Disord. 2014;24(12):1068-72.

3. Alrohaif H, Pogoryelova O, Al-Ajmi A, Aljeryan LA, Alrashidi NH, Alefasi SA, et al. GNE myopathy in the bedouin population of Kuwait: genetics, prevalence, and clinical description. Muscle Nerve. 2018:58(5):700-7.

4. Carrillo N, Malicdan MC, Huizing M. GNE myopathy: etiology, diagnosis, and therapeutic challenges. Neurotherapeutics. 2018;15(4):900-14.

5. Barel O, Kogan E, Sadeh M, Kol N, Nayschool O, Benninger F. Abdominal muscle weakness as a presenting symptom in GNE myopathy. J Clin Neurosci. 2019;59:316-17.

6. Bhattacharya S, Khadilkar SV, Nalini A, Ganapathy A, Mannan AU, Majumder PP, et al. Mutation Spectrum of GNE myopathy in the Indian sub-continent. J Neuromuscul Dis. 2018:5(1):85-92.

7. Chen Y, Xi J, Zhu W, Lin J, Luo S, Yue D, et al. GNE myopathy in Chinese population: hotspot and novel mutations. J Hum Genet. 2019;64(1):11-6.

8. Futterer J, Dalby A, Lowe GC, Johnson B, Simpson MA, Motwani J, et al. Mutation in GNE is associated with severe congenital thrombocytopenia. Blood. 2018;132(17):1855-8.

9. Tomimitsu H, Shimizu J, Ishikawa K, Ohkoshi N, Kanazawa I, Mizusawa H. Distal myopathy with rimmed vacuoles (DMRV): new GNE mutations and splice variant. Neurology. 2004;62(9):1607-10.

10. Mori-Yoshimura M, Monma K, Suzuki N, Aoki M, Kumamoto T, Tanaka K, et al. Heterozygous UDP-GICNAc 2-epimerase and N-acetylmannosamine kinase domain mutations in the GNE gene result in a less severe GNE myopathy phenotype compared to homozygous $\mathrm{N}$-acetylmannosamine kinase domain mutations. J Neurol Sci. 2012;318(1-2):100-5.
11. Leoyklang P, Class B, Noguchi S, Gahl WA, Carrillo N, Nishino I, et al. Quantification of lectin fluorescence in GNE myopathy muscle biopsies. Muscle Nerve. 2018;58(2):286-92.

12. Keppler OT, Hinderlich S, Langner J, Schwartz-Albiez R, Reutter W, Pawlita M. UDP-GICNAc 2-epimerase: a regulator of cell surface sialylation. Science. 1999;284(5418):1372-6.

13. Gagiannis D, Orthmann A, Danssmann I, Schwarzkopf M, Weidemann W, Horstkorte R. Reduced sialylation status in UDP-N-acetylglucosamine-2epimerase/N-acetylmannosamine kinase (GNE)-deficient mice. Glycoconj J. 2007;24(2-3):125-30.

14. Malicdan MC, Noguchi S, Hayashi YK, Nonaka I, Nishino I. Prophylactic treatment with sialic acid metabolites precludes the development of the myopathic phenotype in the DMRV-hlBM mouse model. Nat Med. 2009; 15(6):690-5.

15. Kitazume S, Imamaki R, Ogawa K, Taniguchi N. Sweet role of platelet endothelial cell adhesion molecule in understanding angiogenesis. Glycobiology. 2014;24(12):1260-4

16. Li R, Hoffmeister KM, Falet H. Glycans and the platelet life cycle. Platelets. 2016;27(6):505-11.

17. Zhen C, Guo F, Fang X, Liu Y, Wang X. A family with distal myopathy with rimmed vacuoles associated with thrombocytopenia. Neurol Sci. 2014;35(9): 1479-81.

18. Revel-Vilk S, Shai E, Turro E, Jahshan N, Hi-Am E, Spectre G, et al. GNE variants causing autosomal recessive macrothrombocytopenia without associated muscle wasting. Blood. 2018;132(17):1851-4.

19. Soule T, Phan C, White C, Resch L, Lacson A, Martens K, et al. GNE myopathy with novel mutations and pronounced Paraspinal muscle atrophy. Front Neurol. 2018;9:942.

20. Wu Y, Yuan L, Guo Y, Lu A, Zheng W, Xu H, et al. Identification of a GNE homozygous mutation in a Han-Chinese family with GNE myopathy. J Cell Mol Med. 2018:22(11):5533-8.

\section{Publisher's Note}

Springer Nature remains neutral with regard to jurisdictional claims in published maps and institutional affiliations.
Ready to submit your research? Choose BMC and benefit from:

- fast, convenient online submission

- thorough peer review by experienced researchers in your field

- rapid publication on acceptance

- support for research data, including large and complex data types

- gold Open Access which fosters wider collaboration and increased citations

- maximum visibility for your research: over $100 \mathrm{M}$ website views per year

At BMC, research is always in progress.

Learn more biomedcentral.com/submissions 